\title{
Differential Effect of Orexin-I and CRF-I Antagonism on Stress Circuits: a fMRI Study in the Rat with the Pharmacological Stressor Yohimbine
}

\author{
Alessandro Gozzi*', Stefano Lepore', Elena Vicentini' ${ }^{2}$, Emilio Merlo-Pich ${ }^{2}$ and Angelo Bifone' \\ IIstituto Italiano di Tecnologia, Center for Neuroscience and Cognitive Systems @UniTn, Rovereto, \& Center for Nanotechnology Innovation \\ @NEST, Pisa, Italy; ${ }^{2}$ GlaxoSmithKline Medicine Research Centre, Via Fleming 4, Verona, Italy
}

\begin{abstract}
Translational approaches to study the neural substrates of stress and assess the mechanistic efficacy of novel anti-anxiety agents necessitate the use of stressors with a similar degree of saliency across species. The alpha- 2 adrenoreceptor antagonist yohimbine represents an attractive experimental tool owing to its well-documented stress-inducing properties in humans and laboratory species. We investigated the neural substrates engaged by yohimbine in the rat brain by using functional magnetic resonance imaging and mapped their modulation by neurotransmitter systems involved in stress responses. Yohimbine elicited a composite pattern of brain activation, highlighting the recruitment of cortico-striato-thalamic regions and extra-hypothalamic stress neurocircuits. This effect was strongly attenuated by the $\alpha$-2-adrenoceptor agonist medetomidine and by the dopamine (DA) D, receptor antagonist SCH23390, thus revealing a primary contribution of both norepinephrine and DA on the neurofunctional cascade elicited by the drug. Pretreatment with the corticotrophin-releasing factor type-I receptor (CRFIR) antagonist CPI54,526 produced a region-dependent inhibition of yohimbine-induced activation in the amygdala, striatum, and cingulate cortex, while the orexin type-I receptor (OXIR) antagonists GSK 059865 robustly inhibited the response in fronto-hippocampal regions as well as in several key components of the extended amygdala. CPI 54,526 and GSK 059865 did not prevent yohimbine-induced plasma corticosterone release, a finding that corroborates a central origin of the effects mapped. Our findings provide novel insight into the brain substrates and neurochemical mediators engaged by the stress-inducing agent yohimbine. The differential pattern of inhibition produced by CRFIR and OXIR antagonists suggests that these two neuropeptide systems can modulate the functional response to stress via distinct central neural pathways.

Neuropsychopharmacology (2013) 38, 2120-2I30; doi:I0.1038/npp.2013.109; published online 5 June 2013
\end{abstract}

Keywords: phMRl; yohimbine; CRF; orexin; dopamine; rat

\section{INTRODUCTION}

Yohimbine is a prototypical $\alpha 2$-adrenoreceptor antagonist that induces activation of ascending noradrenaline (NA) systems (Aghajanian and VanderMaelen 1982). Increased noradrenergic activity has been consistently associated to a variety of stressful events, including acute anxiety-like responses (Glavin 1985). Yohimbine's ability to activate noradrenergic pathways has been widely exploited in preclinical research to induce acute stress in laboratory animals under a variety of behavioral paradigms (Shepard et al, 2004a; Richards et al, 2008; Marinelli et al, 2007; Bremner et al, 1996), and to investigate the neurochemical and neuro-physiological determinants of acute stress (Abercrombie et al, 1988). Importantly, the drug has also

*Correspondence: Dr A Gozzi, Istituto Italiano di Tecnologia, Center for Neuroscience and Cognitive Systems @UniTn, Rovereto, Corso Bettini 3।, 38068 Pisa, Italy, Tel: +39 335353 830, Fax: +39 046 48087।0, E-mail: alessandro.gozzi@iit.it

Received 4 February 2013; revised 22 April 2013; accepted 3 May 2013; accepted article preview online 8 May 2013 been shown to increase peripheral and central noradrenergic activity in humans, an effect that is associated to stress and anxiety symptoms (Charney et al, 1982; Cameron et al, 2000; Holmberg and Gershon, 1961; Bremner et al, 1996). These features suggest the possibility of using yohimbine as a titratable, cross-species probe for translational research of stress. Such an approach has the advantage of employing a stressor that works on identifiable neural pathways, and has the same degree of saliency across species. Moreover, when combined with neuroimaging methods like functional magnetic resonance imaging (fMRI), the use of yohimbine offers the opportunity to identify and spatially resolve the neural circuits underlying acute stress. Such a paradigm would be ideally suited for early clinical investigations of the mechanistic efficacy of novel classes of anti-anxiety agents, especially those targeting neuropeptide receptors ligands, for which the development of PET ligands has been so far unsuccessful.

Initial studies of the substrates modulated by yohimbine in the human brain have demonstrated a significant increase in deoxy-glucose metabolism in several 
brain regions of volunteers receiving the drug, with a predominant involvement of fronto-cortical areas (Bremner et al, 1997). Anxiogenic doses of the drug were also reported to increase regional cerebral blood flow (CBF) in cortical and thalamic areas (Cameron et al, 2000). However, the limited spatial resolution of these neuroimaging techniques prevents a fine-grain description of the specific neuronal pathways recruited by the compound. Moreover, no attempts to dissect the contributions of specific receptors and neurotransmitter systems to the functional response elicited by the drug have been described.

Here, we have used pharmacological fMRI to map the neural pathways modulated by the pharmacological stressor yohimbine in the rat brain and examined how these are modulated by specific neurotransmitter systems. We used microvascular cerebral blood volume (CBV) as a marker of neuro-metabolic activity (Gonzalez et al, 1995; Gozzi et al, 2011a) and performed the studies under controlled anesthetic conditions (Ferrari et al, 2012) and with varying anesthetic doses to exclude major drug-anesthetic interactions. As the effect of yohimbine is thought to involve a primary contribution of ascending NA pathways, we examined the inhibitory effect of pretreatment with the selective alpha-2-adrenoreceptors agonist medetomidine (Carlson et al, 1992). Moreover, we investigated the contribution of the dopamine (DA) system to the downstream functional cascade elicited by yohimbine using the selective $\mathrm{DA}_{1}$ receptor inhibitor $\mathrm{SCH} 22390$, a compound that robustly inhibits the functional response to dopaminergic agents (Dixon et al, 2005). We also examined the contribution of neuropeptide receptor systems involved in adaptive and pathological stress responses. Recent studies have demonstrated a critical contribution of orexin (OX)producing neurons in modulating acute stress (Johnson et al, 2010), and a possible anxiolytic action of agents targeting the OX type-1 receptor (OX1R) (Johnson et al, 2012). We used the potent and selective OX1R receptor antagonist GSK1059865 (Gozzi et al, 2011b) to explore a contribution of OX in the neurofunctional cascade activated by yohimbine. Likewise, corticotropin-releasing factor (CRF) type-1 receptor (CRF1R) antagonists have shown the ability to block some of the behavioral effects of yohimbine (Marinelli et al, 2007). CP154,526, a selective CRF1R antagonist and putative anxiolytic (Seymour et al, 2003), was used to probe the role of CRF in modulating the circuital pathways activated by the yohimbine. Finally, postfMRI plasma corticosterone levels were measured in control and yohimbine-treated animals to obtain an assessment of hypothalamic-pituitary-adrenal axis (HPA) activation, and investigate the modulatory role of CP154,526 and GSK1059865 on peripheral stress effectors.

\section{MATERIALS AND METHODS}

All research involving animals were carried out in accordance with the European directive 86/609/EEC governing animal welfare and protection, which is acknowledged by the Italian Legislative Decree no. 116, 27 January 1992. Animal research protocols were also reviewed and consented to by a local animal care committee.

\section{Drug Formulation}

Yohimbine hydrochloride (Tocris Bioscience, Bristol, UK) was suspended in saline containing $5 \%$ dimethyl sulfoxide (DMSO). Medetomidine, and SCH22390 (Tocris Bioscience) were dissolved in saline. CP154,526 was suspended in water containing $0.5 \%$ hydroxy-propyl-methyl-cellulose (HPMC). GSK1059865 was kindly provided by GlaxoSmithKline and dissolved in 5\% DMSO/1.5\% HPMC/0.15\% SLS (sodium lauryl sulfate) plus $10 \%(\mathrm{w} / \mathrm{v})$ D-mannitol as previously described (Gozzi et al, 2011b).

\section{Pharmacological fMRI}

Sprague-Dawley male rats (Chares River, Como, Italy) weighing 250-350 $\mathrm{g}$ were used. Animal preparation and monitoring have been previously described in previous publications (Gozzi et al, 2011a; Ferrari et al, 2012). Briefly, rats were anesthetized with isoflurane, tracheotomized, and artificially ventilated under neuromuscular blockade. After surgery, the isoflurane level was set to $1.1 \%$ unless otherwise stated (see below, group \#2). The ventilation parameters were adjusted to maintain normocapnic arterial $\mathrm{p}_{\mathrm{a}} \mathrm{CO}_{2}$ levels (Pepelko and Dixon 1975), and $\mathrm{p}_{\mathrm{a}} \mathrm{O}_{2}$ levels higher than $90 \mathrm{~mm} \mathrm{Hg}$ corresponding to $>98 \%$ hemoglobin saturation (Supplementary Table S1). MRI data were acquired using a Bruker Avance 4.7 Tesla system. The MR acquisition comprised $\mathrm{T}_{2}$-weighted anatomical images followed by a time series acquisition $\left(\mathrm{TR}=2700 \mathrm{~ms}, \mathrm{TE}_{\mathrm{eff}}=110 \mathrm{~ms}\right.$, RARE factor 32) with a time resolution of $27 \mathrm{~s}$ per brain volume. Following six reference images, $1.5 \mathrm{ml}$ of Molday Ion (Biopal, Boston, USA), a long half-life suspension of superparamagnetic iron oxide nanoparticles, were injected so that signal changes would reflect CBV (rCBV). Drugs were administered 15-25 min after contrast agent administration.

\section{Experimental Design and Pharmacological Treatments}

Three separate studies were performed using different batches of randomly assigned experimental animals.

\section{Study 1}

(1) Oral pretreatment (p.o.) with $0.5 \%$ HPMC, followed by intravenous (i.v) challenge with yohimbine $(0.75 \mathrm{mg} / \mathrm{kg})$ 1 h later $(n=14)$.

(2) $0.5 \%$ HPMC p.o. followed by i.v. yohimbine challenge $(0.75 \mathrm{mg} / \mathrm{kg}) 1 \mathrm{~h}$ later $(n=6)$. This group of subjects was scanned with a maintenance anesthesia level of $1.6 \%$.

(3) Intraperitoneal (i.p.) pretreatment with medetomidine $(20 \mu \mathrm{g} / \mathrm{kg})$ followed by i.v. yohimbine challenge $(0.75 \mathrm{mg} / \mathrm{kg}) 25 \mathrm{~min}$ later $(n=5)$.

(4) CP-154526 (50 mg/kg) p.o. followed by i.v. yohimbine challenge $(0.75 \mathrm{mg} / \mathrm{kg}) \quad 1 \mathrm{~h}$ later $(n=11)$. All oral pretreatments were performed before surgery and fMRI time series were timed to occur $1 \mathrm{~h}$ before yohimbine challenge.

\section{Study 2}

(1) $\mathrm{SCH} 23390(0.1 \mathrm{mg} / \mathrm{kg})$ i.p. followed by i.v. yohimbine challenge $(0.75 \mathrm{mg} / \mathrm{kg}) 15 \mathrm{~min}$ later $(n=7)$. 
(2) Saline i.p. followed by i.v. yohimbine challenge $(0.75 \mathrm{mg} / \mathrm{kg}) 15 \mathrm{~min}$ later $(n=6)$.

Study 3

(1) GSK1059865 (30 mg/kg) i.p. followed by i.v. yohimbine challenge $(0.75 \mathrm{mg} / \mathrm{kg}) 25 \mathrm{~min}$ later $(n=11)$.

(2) Vehicle i.p. followed by i.v. yohimbine challenge $(0.75 \mathrm{mg} / \mathrm{kg}) 25 \mathrm{~min}$ later $(n=9)$.

(3) A group of control animals receiving an oral pretreatment with HPMC, and a challenge injection with saline $1 \mathrm{~h}$ later was used as baseline control $(n=11)$. Compound doses were chosen based on previous studies. Yohimbine produces robust stress and anxiety-like responses in conscious rats at doses of $0.5-5 \mathrm{mg} / \mathrm{kg}$ (Wada and Fukuda 1991; Eroglu and Guven 1998). Pilot doseresponse fMRI studies showed that the dose chosen ensured robust and reliable responses central (fMRI) and peripheral (eg, corticosterone) responses while leaving peripheral cardiovascular parameters (eg, blood pressure) within physiological range. The pharmacokinetic parameters of CP154,526 have been previously published (Chen et al, 1997). The data show that upon p.o. administration to SD rats, maximal plasma concentration occurred at $1 \mathrm{~h}$ after administration (eg, the time of administration of yohimbine). The dose regimen employed for CP154,526 is effective in several stress models (Seymour et al, 2003) and ensures an estimated CRF-1 receptor occupancy $(\approx 60 \%)$ sufficient to ensure robust anxiolytic effects in rodents (Gutman et al, 2011). A non-hypnotic dose of medetomidine was selected (Carlson et al, 1992) so to minimize potential interactions with the anesthetic. The administration time of medetomidine was based on previous studies showing that the compound has a rapid pharmacokinetics (Bol et al, 1997) and exerts sustained effects upon subcutaneous administration (MacDonald et al, 1988). The dose of SCH23390 used robustly antagonizes the effect of dopaminergic agents (Dixon et al, 2005; Zahrt et al, 1997). The pretreatment regimen used with SCH23390 has been previously reported to produce rapid and sustained exposure in the rat brain (Hietala et al, 1992) while minimizing the cataleptic and cognitive-impairing effects reported at higher doses (Wadenberg 1992). The dose and administration regimen of GSK1059865 are behaviorally effective and ensure maximal brain OX1R receptor occupancy, with negligible occupancy of OX type 2 receptors (Gozzi et al, 2011b; Piccoli et al, 2012). Times of vehicle administration were chosen to match those of the corresponding pharmacological treatments.

\section{Plasma Corticosterone Quantification}

Plasma corticosterone quantification was performed in rats from groups 1, 4, 7, and 9 as previously described (Bertani et al, 2010). Briefly, $80 \mu \mathrm{l}$ blood samples were taken from the cannulated femoral artery at the end of the experiment, $30 \mathrm{~min}$ after yohimbine (groups 1, 4, and 7) or vehicle challenge (group 9). Plasma was obtained by centrifugation at $1800 \mathrm{~g}$ for $15 \mathrm{~min}$ at $4{ }^{\circ} \mathrm{C}$ and split into aliquots in the presence of a protease inhibitor mix a. Samples were stored at $-80^{\circ} \mathrm{C}$ until hormone quantification.
Corticosterone was measured by radioimmunoassay (MP Biomedicals, CA, USA).

\section{Data Analysis}

CBV time series were analyzed as previously described (Gozzi et al, 2011a; Gozzi et al, 2012; Ferrari et al, 2012). Image-based time series analysis was carried out using FSL with $0.7 \mathrm{~mm}$ spatial smoothing and a boxcar regressor as input function. Between-group comparisons were carried out using FSL's FLAME. Z (Gaussianised T/F) statistic images were thresholded using clusters determined by $\mathrm{Z}>1.9$ and a corrected cluster significance threshold of $P=0.01$. VOI time courses following yohimbine or pretreatment administration were extracted using a 3D digital reconstruction of a rat brain atlas. For each VOI time course, the area under the curve (AUC) over a 15-min time window covering the peak rCBV response to yohimbine $(8-22 \mathrm{~min}$ post yohimbine injection) was used as a summary statistic of the effect of pretreatment on yohimbine-induced response. For CP154,526 pretreatemnt (group \#4) and its control (group $\# 1$ ), a 10 -min time window (15-25 min post yohimbine injection) was used to better capture the slightly delayed inhibitory effect seen in rCBV time courses. Statistical significance of differences in average $\mathrm{rCBV}$ between treatment groups was assessed by a one-way ANOVA followed by Fisher's LSD test for multiple comparisons. The effect of CP154,526 on resting CBV (basal CBV, bCBV) was mapped as previously described (Gozzi et al, 2011a). Briefly, bCBV time series were spatially normalized to a stereotaxic template set and signal intensity converted into basal CBV $(\mathrm{bCBV}(\mathrm{t}))$ on a pixel-wise basis. bCBV time series were calculated over a 5-min time window starting $10 \mathrm{~min}$ after contrast agent injection, and averaged time-wise. Voxel-wise group statistics was carried out using FSL using multilevel Bayesian inference and a $Z$ threshold $>1.6$ and a corrected cluster significance threshold $\left(p_{c}\right)$ of 0.01 .

\section{RESULTS}

\section{Functional Response to Yohimbine}

Administration of yohimbine produced a composite pattern of rCBV increases (Figures 1 and 2) in several cortico-limbic areas $\left(Z>2.3, p_{c}<0.01\right)$. Prominent foci of activation were observed in prefrontal, cingulate, orbito-frontal, and retrosplenial cortex, with extension into the motor, visual, parietal-, and temporal-association areas. Significant activation was also found in the caudate putamen, ventromedial and dorsolateral thalamus, and ventral hippocampal area. The pattern also encompassed key regions of the extrahypothalamic stress circuit, among which the central nucleus of the amygdala and the bed nucleus of stria terminals (BNST), the shell of the nucleus accumbens appeared to be particularly well-delineated. The magnitude of yohimbine-induced rCBV response was region-dependent (Figure 3), with the largest signal changes occurring in fronto-cortical areas and in thalamic subregions. Yohimbine administration produced a transient decrease in mean arterial blood pressure (MABP) (Supplementary Figure S1). No direct relationship between the anesthesia depth, and sign and magnitude of the functional response to yohimbine 


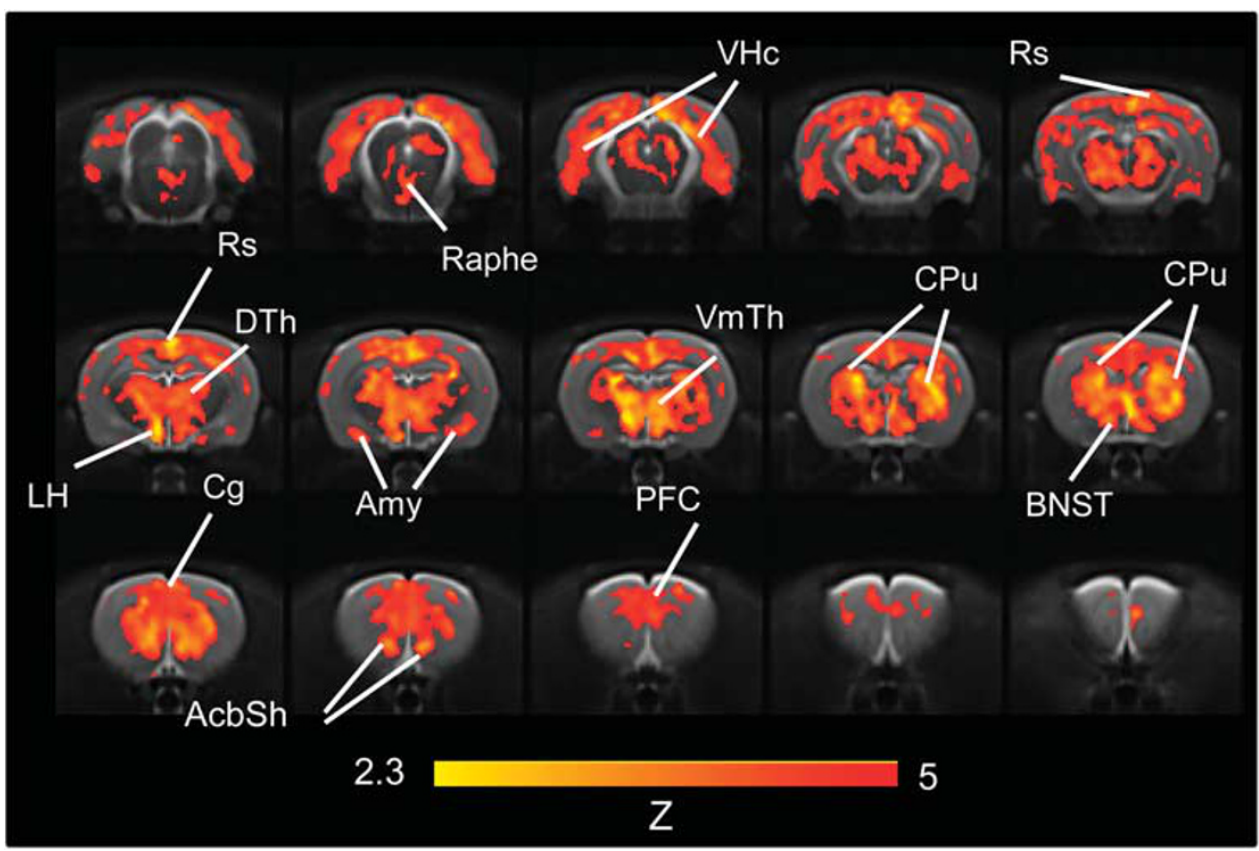

Figure I Anatomical distribution of the brain regions significantly activated by yohimbine in the rat brain $(0.75 \mathrm{mg} / \mathrm{kg}$ i.v., $N=\mid 4$, saline, $N=\mid \mathrm{I})$. Data are arranged rostro-frontally to depict contiguous I-mm-thick coronal slices (AcbSh, nucleus accumbens shell; Amy, amygdala; BNST, bed nucleus of the stria terminalis; Cg, cingulate cortex; Cpu, caudate putamen; DGv, ventral dentate gyrus; DTh, dorsolateral thalamus; LH, lateral hypothalamus; PFC, prefrontal cortex; Rs, retrosplenial cortex; VmTh, ventromedial thalamus; VHc, ventral hippocampus).
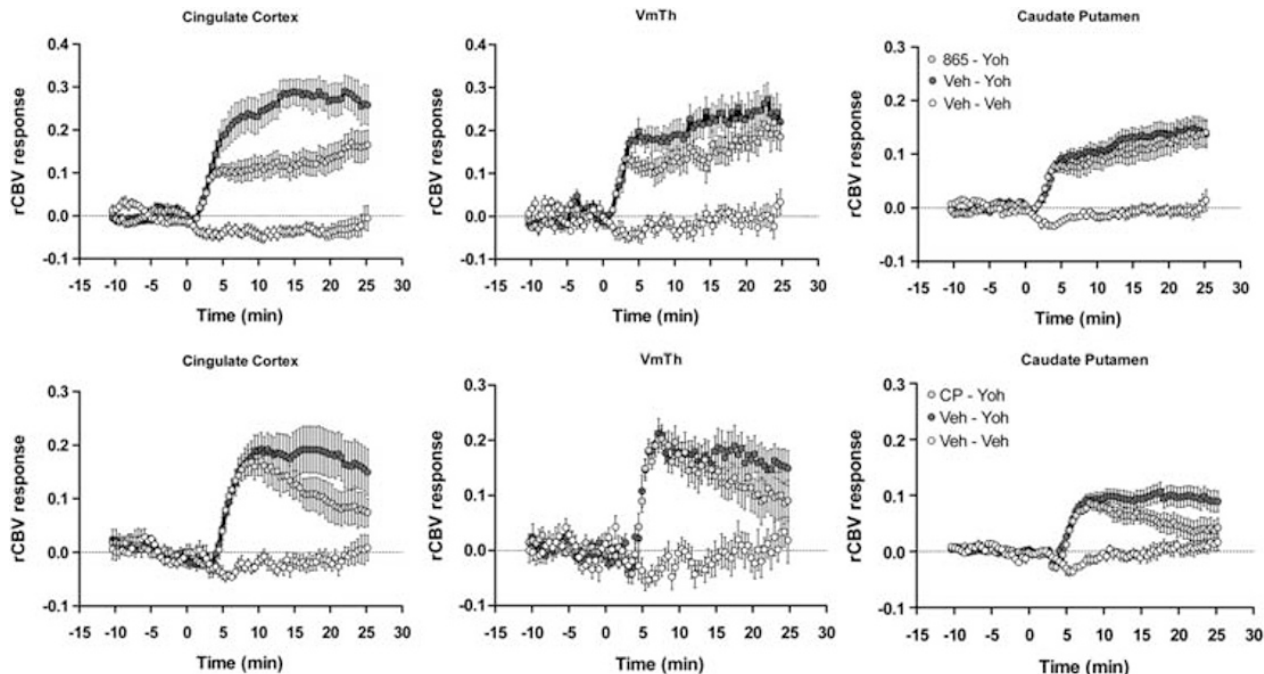

Figure 2 Temporal profile of yohimbine-induced relative cerebral blood volume ( $\mathrm{rCBV}$ ) response in representative anatomical volumes of interest. Yohimbine was injected at time 0. Data are plotted as mean \pm SEM within each group (veh, vehicle; Yoh, yohimbine; CP, CPI54,526; 865, GSKI059865; VmTh, ventromedial thalamus).

was found when the study was replicated using a $50 \%$ increase in anesthetic concentration (Figure 3, $p>0.38$, all regions). Consistent with a deeper anesthetic effect (Imai et al, 1999), lower basal MABP values were observed in animals receiving the higher anesthetic dose (Supplementary Figure S1).

\section{Effect of NA and DA}

Pre-administration of a non-hypnotic and subsedative dose of medetomidine $(20 \mu \mathrm{g} / \mathrm{kg}$ i.p.) produced a robust and generalized attenuation of the rCBV response encompassing all the major target areas of yohimbine action (Figure 3, Supplementary Figure S2). Pretreatment with medetomidine per se produced a transient $\mathrm{rCBV}$ decrease in several cortical and subcortical areas (Supplementary Figure S3), an effect was accompanied by a gradual decrease in MABP that persisted also after yohimbine administration (Supplementary Figure S4). Pre-administration with the selective DA $D_{1}$ receptor antagonist SCH22390 strongly inhibited the functional response to yohimbine (Figure 3 and Supplementary Figure S5) in all the major cortical and 

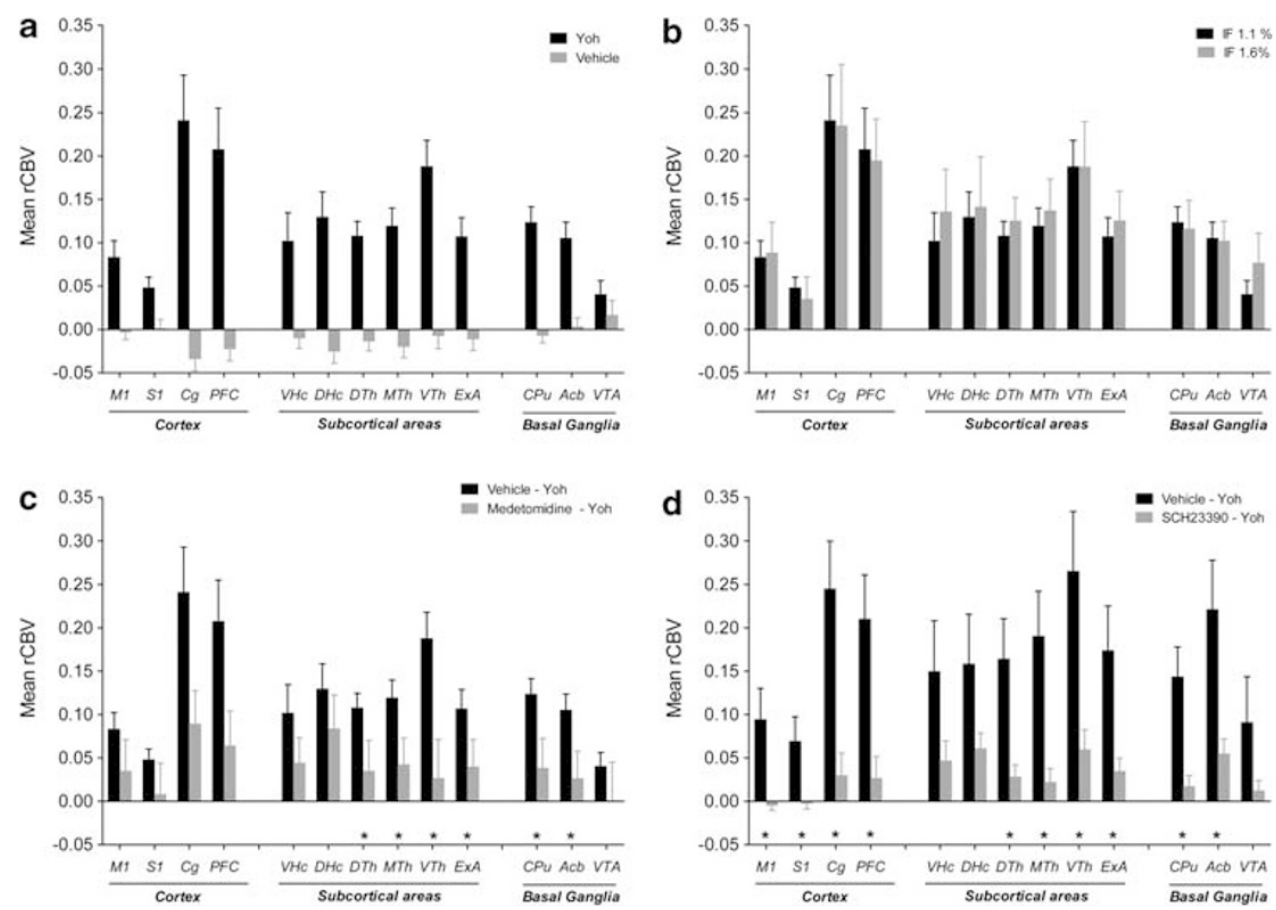

Figure 3 Mean relative cerebral blood volume (rCBV) response elicited by yohimbine in representative anatomical volumes. (a) Mean rCBV response to yohimbine in animals pretreated with vehicle and challenged with yohimbine (Yoh, experimental group I). The effect of pretreatment and challenge with vehicle (saline; experimental group 9) is reported as a baseline reference. The effect of yohimbine was statistically significant ( $p<0.05$, one-way ANOVA, followed by Fisher's LSD test) in all the anatomical volumes with the exception of the VTA. (b) Effect of maintenance anesthesia level on the rCBV response produced by yohimbine. Subjects were pretreated with vehicle, and the rCBV response to yohimbine imaged under isoflurane (IF) anesthesia levels of $1.1 \%$ (experimental group I) or $1.6 \%$ (experimental group 2). No statistically different effect was observed in any of the regions examined. (c) Effect of pretreatment with medetomidine (experimental group 3). (d) Effect of pretreatment with the DA DI antagonist SCH23390 (experimental group 4). ${ }^{*} p<0.05$ vs control group (Vehicle-Yoh) one-way ANOVA, followed by Fisher's LSD test (Acb, nucleus accumbens; Cg, cingulate cortex; CPu, caudate putamen; DHc, dorsal hippocampus; DTh, dorsolateral thalamus; ExA, extended amygdala; MTh, midline thalamus; MI, motor cortex; PFC, prefrontal cortex; Rs, retrosplenial cortex; SI, primary somatosensory cortex;VHc, ventral hippocampus; VTh, ventromedial thalamus; VTA, ventral tegmental area).

subcortical areas activated by yohimbine $\left(Z>1.6, p_{\mathrm{c}}<0.01\right.$, Figure 3 and Supplementary Figure S3). The rCBV changes produced by pre-administration of SCH22390 per se were negligible in all the brain regions examined (Supplementary Figure S6). The drug did not produce major alterations in MABP (Supplementary Figure S7).

\section{Effect of CRF1R Antagonism}

Oral pretreatment with the CRF1R antagonist CP154,526 produced a region-dependent inhibition of the $\mathrm{rCBV}$ response elicited by yohimbine (Figures $2-4$ ). The inhibitory effect presented itself in the form of an attenuated response to that become apparent 5-8 min after yohimbine injection. Image-based statistics highlighted significant foci of response attenuation in the motor, cingulate, retrosplenial, and dorsal prefrontal cortex $\left(Z>1.6 ; p_{c} 0.01\right.$, Figure 3$)$, dorsal portions of the caudate putamen, and in the amygdala. Administration of CP154,526 per se did not produce significant alterations in basal CBV (bCBV; $Z>1.6, p_{\mathrm{c}}=0.01$ ). Linear regression did not highlight any significant correlation between $\mathrm{bCBV}$ and subsequent yohimbine-induced rCBV response (expressed as $\mathrm{AUC}_{10-25} \mathrm{~min}$ ) in any of the VOIs examined ( $p>0.28$, all regions). The administration of CP15426 was accompanied by a slight $(\sim 10 \mathrm{~mm} \mathrm{Hg})$ reduction in MABP (Supplementary Figure S1).

\section{Effect of OX1R Antagonism}

Intraperitoneal administration of GSK1059865 produced a region-dependent inhibition of yohimbine-induced rCBV response (Figures 2, 3, and 5). Significant foci of response attenuation were observed in the nucleus accumbens, septum, dorsal thalamus, amygdala, ventral hippocampus, orbito-frontal, prefrontal, insular, cingulate retrosplenial, and piriform cortex $\left(Z>1.6 ; p_{c} 0.01\right.$, Figure 5). The inhibitory effect was larger and more prominent in fronto-cortical areas with respect to all the other attenuated regions. The administration of GSK1059865 per se produced a weak rCBV increase in several brain regions (Supplementary Figure S8). No significant correlation was found between the magnitude of $\mathrm{rCBV}$ response produced by i.p. pretreatemnt $\left(\mathrm{AUC}_{1-25} \mathrm{~min}\right)$ and the subsequent rCBV response to yohimbine $\left(\mathrm{AUC}_{10-25} \mathrm{~min}\right)$ in any of the regions examined ( $p>0.17$ all VOIs). GSK1059865-pretreated animals exhibited slightly higher baseline MABP values than controls (Supplementary Figure S7). No significant correlation was found between pre-yohimbine MABP values and the magnitude of the $\mathrm{rCBV}$ response produced by the yohimbine challenge $\left(\mathrm{AUC}_{1-25} \mathrm{~min}\right)$ in any of the regions examined ( $p>0.52$ all VOIs). Postyohimbine MABP levels were comparable between groups (Supplementary Figure S1). 

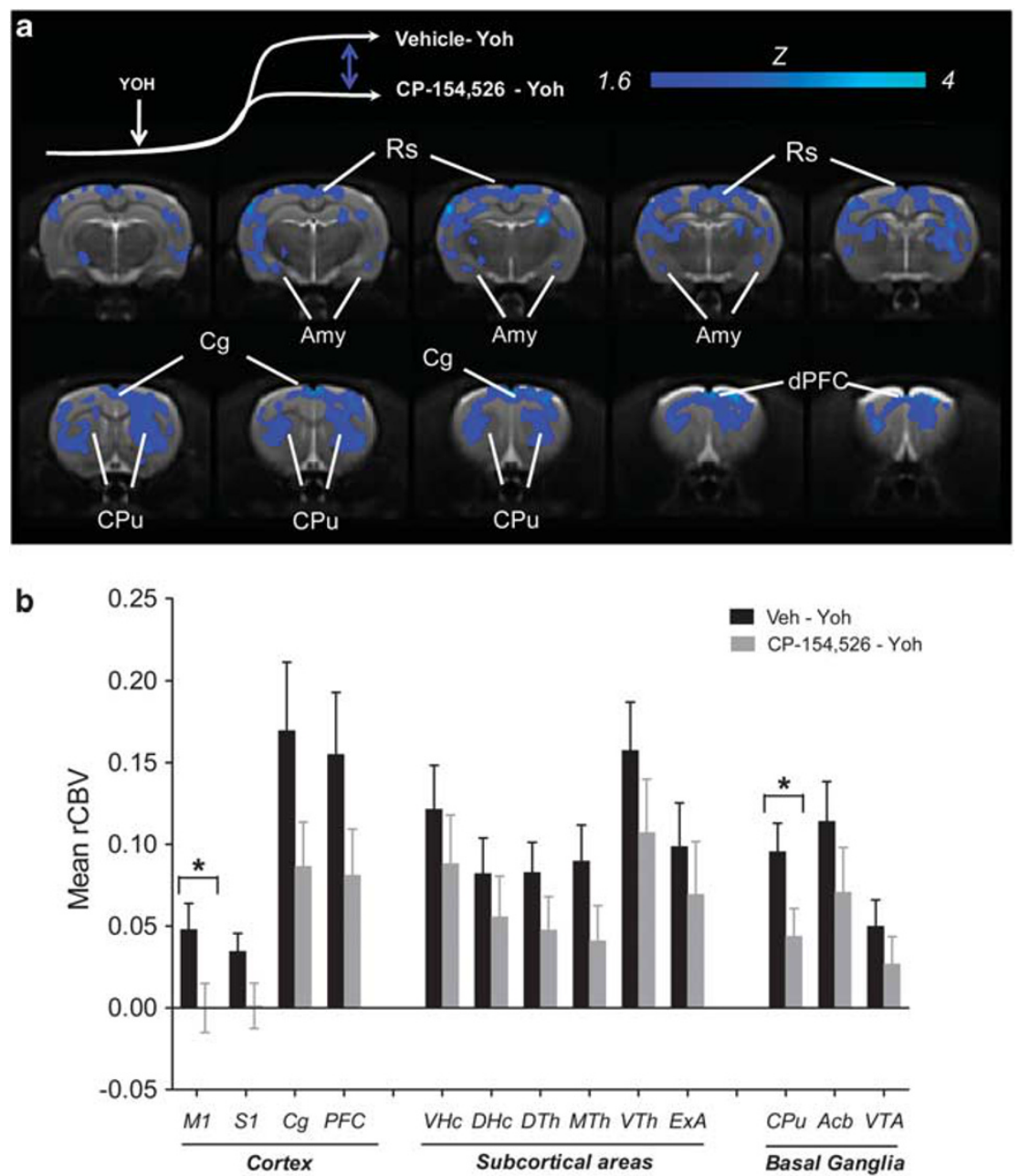

Figure 4 (a) Anatomical distribution of the brain regions exhibiting a significantly reduced $\left(z>1.6, p_{c}=0.01\right)$ rCBV response to yohimbine in animals pretreated with CPI54,526 with respect vehicle. Yoh, yohimbine. (b) Mean relative cerebral blood volume ( $r C B V$ ) response elicited by yohimbine in representative volumes of interest as a function of pretreatment. * $<0.05$ vs control group (Vehicle-Yoh) one-way ANOVA, followed by Fisher's LSD test (Amy, amygdala; $\mathrm{Cg}$, cingulate cortex; CPu, caudate putamen; dPFC, dorsal prefrontal cortex; Rs, retrosplenial cortex).

\section{Plasma Corticosterone Measurements}

Plasma corticosterone levels were measured at the end of the fMRI experiment in control animals (group 9) and in subjects challenged with Yohimbine (groups 1, 4, and 7). Basal corticosterone levels at the end of the experiment were in excellent agreement with previous measurements in conscious animals at points of the circadian cycle similar to those at which the experiments were performed (eg, 6-8 h into the light phase, cf. Bertani et al, 2010; Atkinson and Waddell 1997) thus ruling out a major contribution of unspecific stressful effects of the anesthetic or animal preparation procedure. Yohimbine administration was associated to a significant increase in plasma corticosterone levels $(p<0.05$, one-way ANOVA; all groups). Pretreatment with CP154,526 or GSK1059865 did not to significantly alter yohimbineinduced corticosterone release ( $p>0.22$, all groups).

\section{DISCUSSION}

Consistent with the established role of distributed networks in mediating acute response to stress, our fMRI study revealed the activation of a composite pattern of regions implicated in stress upon acute administration of the pharmacological stressor yohimbine. The extra-hypothalamic stress circuit (ie, brain stem nuclei, the hypothalamus, the amygdala, the BNST, and the shell of the nucleus accumbens) appeared to be particularly well delineated in the frontal portions of the brain, including a clear involvement of the shell of the nucleus accumbens. The latter finding is in agreement with the neuroanatomical definition of 'extended amygdala', a meta-structure that has a key role in the heightened stress sensitivity and negative emotional states associated with drug addiction and stressinduced facilitation of anxiety (Koob and Kreek, 2007). The robust activation of prefrontal and orbito-frontal areas, together with the predominant involvement of the thalamus, ventral pallidum, and caudate putamen define a corticothalamo-striatal network reminiscent of a loop that appears to be dysregulated in compulsive states (Shin and Liberzon, 2009). This finding is interesting in the light of the ability of the compound to reliably and robustly reinstate compulsive behaviors such as food (Ghitza et al, 2007; Nair et al, 2009; Cifani et al, 2012) and drug seeking (Feltenstein and See 


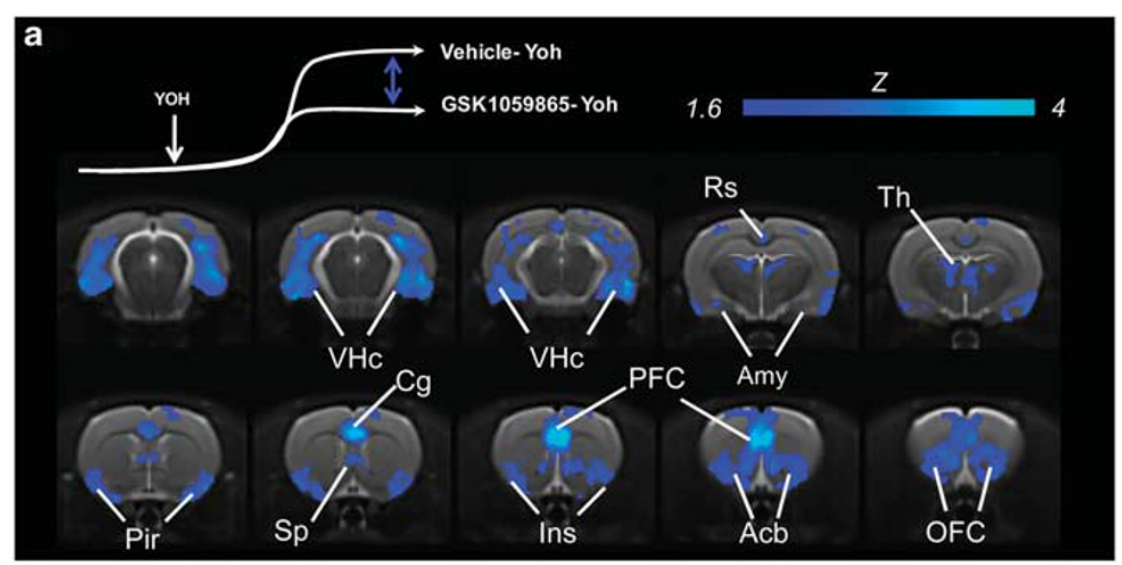

b

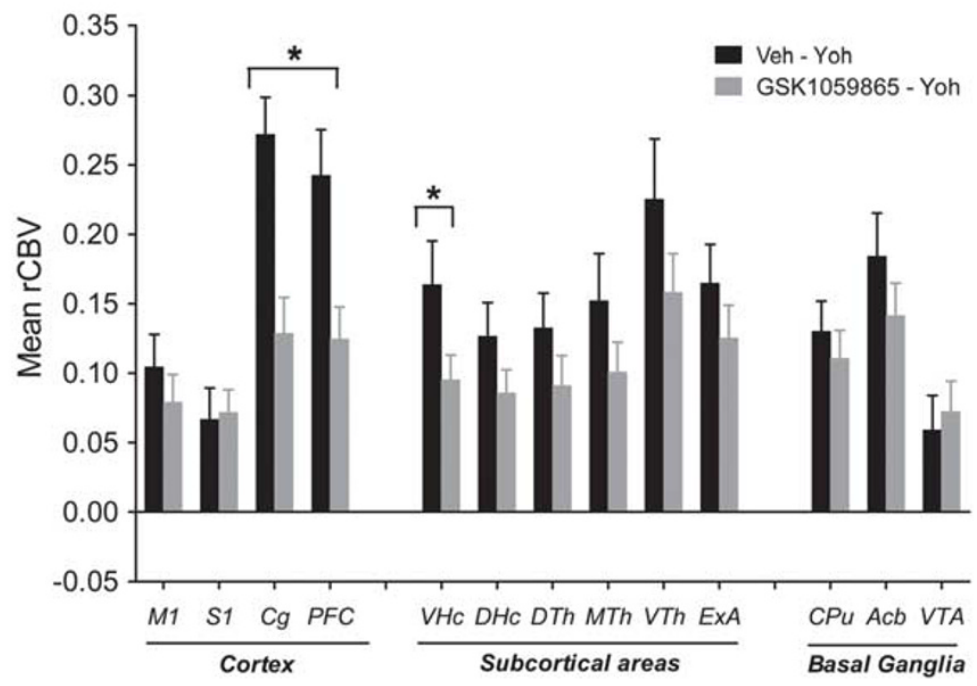

Figure 5 (a) Anatomical distribution of the brain regions exhibiting a significantly reduced $\left(z>1.6, p_{c}=0.0 \mathrm{l}\right)$ rCBV response to yohimbine in animals pretreated with GSKI059865 with respect vehicle. Yoh, yohimbine. (b) Mean relative cerebral blood volume (rCBV) response elicited by yohimbine in representative volumes of interest as a function of pretreatment. ${ }^{*} p<0.05$ vs control group (Vehicle-Yoh) one-way ANOVA, followed by Fisher's LSD test (Acb, nucleus accumbens; Cg, cingulate cortex; Ins, insular cortex; OFC, orbito-frontal cortex; Pir, piriform cortex; PFC, prefrontal cortex; Rs, retrosplenial cortex; Sp, septum; Th, thalamus; VHc, ventral hippocampus; VTh, ventromedial thalamus; VTA, ventral tegmental area).

2006; Kupferschmidt et al, 2009; Shepard et al, 2004b; Marinelli et al, 2007; Brown et al, 2009; Lee et al, 2004) in animal models of addiction. Our imaging results provide for the first time a systems level description of the central substrates recruited by this important translation tool in the study of stress-induced relapse. The drug also elicited a significant activation of the HPA, a finding consistent with the stress-inducing properties of the compund and the results of previous animal (Marinelli et al, 2007) and human studies (Hedner et al, 1992).

The fact that our experiments were performed in anesthetized animals raises the question as to whether the observed effects truly reflect the effect of yohimbine in conscious states. We chose to map the effect of yohimbine in animals under light isoflurane anesthesia, as previous research showed the lack of significant pharmacological interactions between the two drugs (de Wolff et al, 1999). In order to confirm this, we repeated the functional mapping in a cohort of subjects using deeper isoflurane anesthesia (from $1.1 \%$ to $1.6 \%$, ca. $+50 \%$ ). The results we obtained strongly argue against a major interference of anesthesia on the functional changes mapped, because both the distribution and the magnitude of the functional effects mapped with the two anesthetic levels were comparable. Previous studies performed in our lab showed that when druganesthetic interactions do occur, small changes $(20 \%)$ in anesthetic dose are sufficient to dramatically alter the sign and spatial distribution of the fMRI response (Gozzi et al, 2008). Importantly, Fos immunoreactivity studies in conscious animals challenged with yohimbine (Singewald et al, 2003) or exposed to environmentally evoked stress and fear (Beck and Fibiger, 1995; Duncan et al, 1996) revealed similarly robust and widespread functional responses in the vast majority of regions identified in the present study. The spatial correspondence between cellular markers of activation (ie Fos) and the haemodynamic readout used here strongly corroborates a neural (rather than vascular) origin of the functional changes, and defines our imaging results as a plausible substrate of the stress-inducing effect of yohimbine in freely behaving animals. Importantly, the pattern of activation identified is also in agreement with the result of human neuroimaging studies. Widespread 


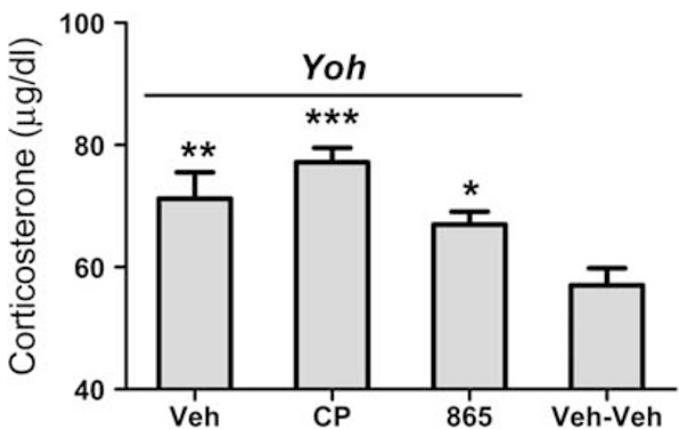

Figure 6 Plasma corticosterone levels as measured at the end of the fMRI session (30 min after yohimbine challenge). Basal corticosterone levels from a group of animals challenged and pretreated with vehicle (group 9, Veh-Veh) are reported for comparison $* p<0.05$ vs Veh-Yoh (group \#I); *** $<0.05$ vs Veh-Yoh (group \#I); **** $<<0.0$ I vs Veh-Yoh (group \#I) (Veh, vehicle; Yoh, yohimbine; CP, CPI54,526; GSK, GSKI059865; VmTh, ventromedial thalamus).

metabolic increases, with the predominant involvement of fronto-cortical areas, was observed in fluoro-deoxy-glucose PET studies with yohimbine in human volunteers (Bremner, 1997). Anxiogenic doses of the drug were also reported to increase regional $\mathrm{CBF}$ in cortical and thalamic areas (Cameron et al, 2000), an effect that was, however, accompanied by a global reduction in CBF across the brain. While this latter finding may appear at odds with the sign of the CBV changes mapped here, it should be noted that in Cameron's study yohimbine produced significant reductions in arterial $\mathrm{pCO}_{2}$ as a result of hyperventilation. As pointed out by the authors, the CBF reduction is thus likely to be an unspecific consequence of the sharp $\mathrm{pCO}_{2}$ decrease observed upon yohimbine administration. In the present study, the use of artificial ventilation prevented abrupt arterial blood gas alterations consequent to hyperventilation and enabled us to map increased $\mathrm{rCBV}$ responses to yohimbine challenge in agreement with the result of human metabolic measurements (Bremner, 1997) and regional CBF mapping (Cameron et al, 2000). Collectively, the overall agreement between our findings and the limited clinical data available is encouraging, and supports the translational relevance of the imaging approach described here.

\section{Role of NE and DA}

Yohimbine's action at $\alpha 2$-adrenoreceptors activates ascending NA systems by blocking $\alpha 2$-autoreceptors (Abercrombie et al, 1988). In the present work, pretreatment with a nonhypnotic dose of the selective and potent $\alpha 2$-adrenoreceptors potently inhibited the functional response to the drug, a result consistent with results of neurochemical and behavioral studies (Johnston and File, 1989; Abercrombie et al, 1988). The robust inhibition observed despite the use of a low dose of medetomidine corroborates a primary contribution of noradrenergic systems to the functional cascade elicited by the drug. However, the widespread effects of yohimbine are likely to involve the contribution of additional neurotransmitters systems that are known to modulate (and to be modulated by) noradrenergic responses. For example, both $\mathrm{DA}$ and NA appear to be released by most NA terminals (Devoto et al, 2004) and several studies support the existence of a direct action of DA on different subtypes of adrenergic receptors and vice versa (Gresch et al, 1995; Cornil and Ball, 2008). In an attempt to dissect the contribution of DA to the complex functional response elicited by yohimbine, we imaged the modulatory effect produced by pretreatment of the selective D1 antagonist SCH223390. Interestingly, pretreatment with SCH22390 produced a widespread inhibition of yohimbine-induced activation, suggestive of a permissive role of DA neurotransmission in the haemodynamic cascade elicited by the drug. The effect is unlikely to reflect an unspecific inhibitory role of DA D1 receptors on neurovascular coupling, because the same dose of SCH22390 did not alter the fMRI response elicited by glutamatergic agents under the same experimental conditions of the present study (Gozzi et al, 2010b). Although the exact behavioral and neuro-physiological significance of the inhibitory effect of SCH22390 remain to be determined, our findings are in keeping with the emerging role of DA in mediating some the effects of yohimbine. For example, SCH22390 has been recently reported to block yohimbine-induced reinstatement of cocaine and high-fat food seeking (Brown et al, 2012; Nair et al, 2011). The compound also prevented yohimbine-induced functional activation as measured with Fos immunoreactivity in the dorsal prefrontal cortex (Nair et al, 2011). Based on our imaging results, we speculate that the inhibitory effects reported in the two behavioral works could in fact reflect a generalized attenuation of the functional effects of yohimbine produced by DA D1 antagonism. Additional experiments that may employ D1knockout mice lines could help elucidate the functional and behavioral contribution of noradrenergic and dopaminergic mechanisms to the stress-inducing effect of the drug.

\section{Effect of CRF1R Antagonism}

Substantial evidence points at a crucial contribution of endogenous CRF systems as a key mediator of adaptive response to stress, an effect involving reciprocal interactions with noradrenergic systems (Dunn and Swiergiel, 2008). Consistent with this, CRF1R antagonists have been shown to antagonize behavioral responses to stress (Kehne and Cain, 2010; Gutman et al, 2011), including the reinstatement effect of yohimbine on drug seeking (Marinelli et al, 2007). We used the selective CRF1R antagonist CP154,526 to probe a downstream contribution of CRF systems in the circuital pathways activated by the yohimbine, and observed an inhibitory effect in the amygdala, cortex, and corpus striatum. The inhibitory effect in the amygdala, a region pivotally involved in behavioral response to fear (Gozzi et al, 2010a), and a major extra-hypothalamic source of CRF-containing neurons (Van Pett et al, 2000), represents a plausible substrate for the anxiolytic effect exhibited by CRF1R antagonists. Acute stress has been reported to induce increased extracellular CRF in this region (Pich et al, 1995) and administration of CRF1R antagonists in the amygdala attenuates anxiety-like behavior (Rassnick et al, 1993). Similarly, the presence of cortical inhibition is in agreement with the high CRF1R density observed in the rat neocortex (Van Pett et al, 2000) and the ability of CRF1R antagonists to reduce stressinduced NA release in cortical areas (Emoto et al, 1993). 
Among the cortical areas showing foci of inhibition, the preferential involvement of cingulate and dorsal prefrontal areas is of interest in the light of the established contribution of these areas in emotional processing and their hyperactivity in different forms of stress and anxiety (Shin and Liberzon, 2009). The robust striatal modulation observed with CRF1R antagonism was unexpected, as this region has not been classically considered a major site of CRF action despite its abundant CRF1R density (Van Pett et al, 2000). Interestingly, recent neuroimaging data in humans revealed a focal and dose-dependent decrease in striatal metabolism upon administration of a selective CRF1R antagonism (Schmidt et al, 2010). Together with these human findings, our data suggest an unforeseen contribution of the caudate putamen as a substrate for some of the pharmacological effect of this class of compounds. Importantly, antagonism at CRF1R failed to inhibit yohimbine-induced corticosterone release as seen with post-fMRI plasma measurements. This finding is consistent with the results of behavioral studies (Marinelli et al, 2007) and corroborates a central (extra-hypothalamic) origin of the fMRI modulation pattern observed with CP154,526.

\section{Effect of OX1R Antagonism}

The neuroanatomical location and pivotal role played by OX in arousal and wakefulness are in agreement with a role of this neuropeptide system in acute and/or pathological stress. A critical contribution of OX-producing neurons in determining acute stress and panic responses has been recently described (Johnson et al, 2010). Moreover, OX1R receptor antagonists have been reported to inhibit yohimbine-induced reinstatement of drug seeking (Richards et al, 2008; Boutrel, 2005), and the anxiogenic response to the $\mathrm{GABA}_{\mathrm{A}}$ modulator FG-7142 (Johnson et al, 2012), a finding associated to a reduced fos activation in the hypothalamus and amygdala. Our imaging results with the selective OX1R antagonist GSK1059865 add to these findings, by providing evidence of a robust functional inhibition of yohimbineinduced activation in multiple brain regions involved in stress and anxiety. Hyperactivity of the amygdala, insular cortex, anterior cingulated, and prefrontal areas has been consistently observed in anxiety and fear states (Engel et al, 2009; Shin and Liberzon, 2009), and pharmacological inhibition of the functional activity in these areas has been associated with anxiolytic responses (Simmons et al, 2009; Arce et al, 2008). Reduced hippocampal and para-hippocampal perfusion has also been associated to the anxiolytic effect of cannabidiol (Crippa et al, 2011). The modulatory effect of GSK1059865 in DA-innervated terminals such as the nucleus accumbens and the orbito-frontal cortex is in good agreement with previous evidence of a pivotal modulatory contribution of the OX1R on the mesolimbic dopaminergic activity (Gozzi et al, 2011b). In contrast with the recent results of Johnson et al (2012), no functional inhibition in hypothalamic regions was observed with GSK1059865, a finding that, however, could reflect the use of different functional readouts (fos $v s$ fMRI) and anxiogenic drug (FG-7142 vs yohimbine). Collectively, the inhibitory effect identified with GSK1059865 is consistent with the emerging role of the OX1R as a mediator of acute stress responses, an effect that may involve reciprocal modulation with CRF and noradrenergic systems (Boutrel 2005). The different pattern of modulation observed with CRF1R and OX1R antagonists suggests that the two systems can ultimately modulate divergent neuronal pathways. Importantly, both drugs did not significantly alter yohimbine-induced corticosterone release, a finding consistent with previous studies (Marinelli et al, 2007; Laorden et al, 2012) that corroborates a central origin of the fMRI modulation pattern observed. However, whether the inhibitory action exerted by GSK1059865 on the fMRI response to yohimbine would effectively translate into a behaviorally relevant 'anxiolytic' effect remains to be determined. The availability of selective pharmacological tools like GSK1059865 is expected to expedite the investigation of the role of OX1R across different domains of translational neuroscience, including the research for novel pharmacological targets to tackle stress and anxiety.

In conclusion, we show that the anxiogenic drug yohimbine robustly activates a composite cortico-limbic network of regions in the rat brain. We also provided evidence of a primary contribution of NA and DA in the functional cascade elicited by the compound. Finally, we show that drugs targeting CRF-1 or OX1 receptors can region-dependently attenuate yohimbine-induced activation via the involvement of distinct neuronal circuits comprising regions involved in stress responses. We propose the use of pharmacological stressors in combination with spatially resolved neuroimaging techniques like fMRI as a translational paradigm for testing the mechanistic efficacy of novel therapeutic agents targeting stress circuits and their effectors.

\section{FUNDING AND DISCLOSURE}

The authors declare no conflict of interest.

\section{ACKNOWLEDGEMENTS}

We would like to thank Alberto Galbusera for his excellent technical support. The authors declare that, except for income received from their primary employer, no financial support or compensation has been received from any individual or corporate entity over the past 3 years for research or professional service and there are no personal financial holdings that could be perceived as constituting a potential conflict of interest.

\section{REFERENCES}

Abercrombie ED, Keller RW Jr, Zigmond MJ (1988). Characterization of hippocampal norepinephrine release as measured by microdialysis perfusion: pharmacological and behavioral studies. Neuroscience 27: 897-904.

Aghajanian GK, VanderMaelen CP (1982). Alpha 2-adrenoceptormediated hyperpolarization of locus coeruleus neurons: intracellular studies in vivo. Science 215: 1394-1396.

Arce E, Simmons A, Lovero K, Stein M, Paulus M (2008). Escitalopram effects on insula and amygdala BOLD activation during emotional processing. Psychopharmacology 196: 661-672. Atkinson HC, Waddell BJ (1997). Circadian variation in basal plasma corticosterone and adrenocorticotropin in the rat: sexual 
dimorphism and changes across the Estrous Cycle. Endocrinology 138: 3842-3848.

Beck CH, Fibiger HC (1995). Conditioned fear-induced changes in behavior and in the expression of the immediate early gene c-fos: with and without diazepam pretreatment. J Neurosci 15: 709-720.

Bertani S, Carboni L, Criano A, Michielin F, Mangiarini L, Vicentini E (2010). Circadian profile of peripheral hormone levels in Sprague-Dawley Rats and in common marmosets (Callithrix jacchus). In Vivo 24: 827-836.

Bol CJJG, Danhof M, Stanski DR, Mandema JW (1997). Pharmacokinetic-pharmacodynamic characterization of the cardiovascular, hypnotic, EEG and ventilatory responses to dexmedetomidine in the rat. J Pharmacol Exp Ther 283: 1051-1058.

Boutrel B (2005). Role for hypocretin in mediating stress-induced reinstatement of cocaine-seeking behavior. Proc Natl Acad Sci USA 102: 19168-19173.

Bremner J (1997). POsitron emission tomography measurement of cerebral metabolic correlates of yohimbine administration in combat-related posttraumatic stress disorder. Arch Gen Psychiatry 54: 246-254.

Bremner JD, Innis RB, Ng CK, Staib LH, Salomon RM, Bronen RA et al (1997). Positron emission tomography measurement of cerebral metabolic correlates of yohimbine administration in combat-related posttraumatic stress disorder. Arch Gen Psychiatry 54: 246-254.

Bremner JD, Krystal JH, Southwick SM, Charney DS (1996). Noradrenergic mechanisms in stress and anxiety: I. Preclinical studies. Synapse 23: 28-38.

Brown ZJ, Kupferschmidt DA, Erb S (2012). Reinstatement of cocaine seeking in rats by the pharmacological stressors, corticotropin-releasing factor and yohimbine: role for D1/5 dopamine receptors. Psychopharmacology 224: 431-440.

Brown ZJ, Tribe E, D'souza NA, Erb S (2009). Interaction between noradrenaline and corticotrophin-releasing factor in the reinstatement of cocaine seeking in the rat. Psychopharmacology 203: $121-130$.

Cameron OG, Zubieta JK, Grunhaus L, Minoshima S (2000). Effects of yohimbine on cerebral blood flow, symptoms, and physiological functions in humans. Psychosom Med 62: 549-559.

Carlson S, Tanila H, Rama P, Mecke E, Pertovaara A (1992). Effects of medetomidine, an alpha-2 adrenoceptor agonist, and atipamezole, an alpha-2 antagonist, on spatial memory performance in adult and aged rats. Behav Neural Biol 58: 113-119.

Charney DS, Heninger GR, Sternberg DE (1982). Assessment of alpha 2 adrenergic autoreceptor function in humans: effects of oral yohimbine. Life Sci 30: 2033-2041.

Chen YL, Mansbach RS, Winter SM, Brooks E, Collins J, Corman ML et al (1997). Synthesis and oral efficacy of a 4-(butylethylamino)pyrrolo[2,3-d]pyrimidine: $\Gamma$ Çë A centrally active corticotropin-releasing factor1 receptor antagonist. J Med Chem 40: 1749-1754.

Cifani C, Koya E, Navarre BM, Calu DJ, Baumann MH, Marchant NJ et al (2012). Medial prefrontal cortex neuronal activation and synaptic alterations after stress-induced reinstatement of palatable food seeking: a study using c-fos-GFP transgenic female rats. J Neurosci 32: 8480-8490.

Cornil CA, Ball GF (2008). Interplay among catecholamine systems: dopamine binds to + 2-adrenergic receptors in birds and mammals. J Comp Neurol 511: 610-627.

Crippa JA, Derenusson GN, Ferrari TB, Wichert-Ana L, Duran FL, Martin-Santos R et al (2011). Neural basis of anxiolytic effects of cannabidiol (CBD) in generalized social anxiety disorder: a preliminary report. J Psychopharmacol 25: 121-130.

de Wolff MH, Leather HA, Wouters PF (1999). Effects of tramadol on minimum alveolar concentration (MAC) of isoflurane in rats. Br J Anaesth 83: 780-783.
Devoto P, Flore G, Pira L, Longu G, Gessa GL (2004). Alpha2adrenoceptor mediated co-release of dopamine and noradrenaline from noradrenergic neurons in the cerebral cortex. J Neurochem 88: 1003-1009.

Dixon AL, Prior M, Morris PM, Shah YB, Joseph MH, Young AMJ (2005). Dopamine antagonist modulation of amphetamine response as detected using pharmacological MRI. Neuropharmacology 48: 236-245.

Duncan GE, Knapp DJ, Breese GR (1996). Neuroanatomical characterization of Fos induction in rat behavioral models of anxiety. Brain Res 713: 79-91.

Dunn AJ, Swiergiel AH (2008). The role of corticotropin-releasing factor and noradrenaline in stress-related responses, and the inter-relationships between the two systems. Eur J Pharmacol 583: 186-193.

Emoto H, Koga C, Ishii H, Yokoo H, Yoshida M, Tanaka M (1993). A CRF antagonist attenuates stress-induced increases in NA turnover in extended brain regions in rats. Brain Res 627: 171-176.

Engel K, Bandelow B, Gruber O, Wedekind D (2009). Neuroimaging in anxiety disorders. J Neural Transm 116: 703-716.

Eroglu L, Guven O (1998). The effects of moclobemide on the yohimbine-induced anxiogenic action in the elevated plus-maze. Pharmacol Res 37: 137-143.

Feltenstein MW, See RE (2006). Potentiation of cue-induced reinstatement of cocaine-seeking in rats by the anxiogenic drug yohimbine. Behav Brain Res 174: 1-8.

Ferrari L, Turrini G, Crestan V, Bertani S, Cristofori P, Bifone A et al (2012). A robust experimental protocol for pharmacological fMRI in rats and mice. J Neurosci Methods 204: 9-18.

Ghitza UE, Nair SG, Golden SA, Gray SM, Uejima JL, Bossert JM et al (2007). Peptide YY3-36 decreases reinstatement of high-fat food seeking during dieting in a rat relapse model. J Neurosci 27: $11522-11532$

Glavin GB (1985). Stress and brain noradrenaline: a review. Neurosci Biobehav Rev 9: 233-243.

Gonzalez RG, Fischman AJ, Guimaraes AR, Carr CA, Stern CE, Halpern EF et al (1995). Functional MR in the evaluation of dementia: correlation of abnormal dynamic cerebral blood volume measurements with changes in cerebral metabolism on positron emission tomography with fludeoxyglucose F 18. AJNR Am J Neuroradiol 16: 1763-1770.

Gozzi A, Apar J, Giovanelli A, Bertollini C, Crestan V, Schwarz AJ et al (2010a). A neural switch for active and passive fear. Neuron 67: 656-666.

Gozzi A, Colavito V, Seke Etet PF, Montanari D, Fiorini S, Tambalo $S$ et al (2012). Modulation of fronto-cortical activity by modafinil: a functional imaging and Fos study in the rat. Neuropsychopharmacology 37: 822-837.

Gozzi A, Crestan V, Turrini G, Clemens M, Bifone A (2010b). Antagonism at serotonin 5-HT2A receptors modulates functional activity of frontohippocampal circuit. Psychopharmacology 209: 37-50.

Gozzi A, Schwarz AJ, Reese T, Crestan V, Bifone A (2008). Druganaesthetic interaction in phMRI: the case of the pyschotomimetic agent phencyclidine. Magn Reson Imag 26: 999-1006.

Gozzi A, Tessari M, Dacome L, Agosta F, Lepore S, Lanzoni A et al (2011a). Neuroimaging evidence of altered fronto-cortical and striatal function after prolonged cocaine self-administration in the rat. Neuropsychopharmacology 36: 2431-2440.

Gozzi A, Turrini G, Piccoli L, Massagrande M, Amantini D, Antolini $\mathrm{M}$ et al (2011b). Functional magnetic resonance imaging reveals different neural substrates for the effects of orexin-1 and orexin-2 receptor antagonists. PLoS One 6: e16406.

Gresch PJ, Sved AF, Zigmond MJ, Finlay JM (1995) Local influence of endogenous norepinephrine on extracellular dopamine in rat medial prefrontal cortex. J Neurochem 65: 111-116.

Gutman DA, Owens MJ, Thrivikraman KV, Nemeroff CB (2011). Persistent anxiolytic affects after chronic administration of the 
CRF1 receptor antagonist R121919 in rats. Neuropharmacology 60: 1135-1141.

Hedner T, Edgar B, Edvinsson L, Hedner J, Persson B, Pettersson A (1992). Yohimbine pharmacokinetics and interaction with the sympathetic nervous system in normal volunteers. Eur J Clin Pharmacol 43: 651-656.

Hietala J, Sepp T, Lappalainen J, Syvlahti E (1992). Quantification of SCH 39166, a novel selective D1 dopamine receptor antagonist, in rat brain and blood. Psychopharmacology 106: 455-458.

Holmberg G, Gershon S (1961). Autonomic and psychic effects of yohimbine hydrochloride. Psychopharmacologia 2: 93-106.

Imai A, Steffey EP, Farver TB, Ilkiw JE (1999). Assessment of isoflurane-induced anesthesia in ferrets and rats. Am J Vet Res 60: $1577-1583$.

Johnson PL, Samuels BC, Fitz SD, Federici LM, Hammes N, Early MC et al (2012). Orexin 1 receptors are a novel target to modulate panic responses and the panic brain network. Physiol Behav 107: 733-742.

Johnson PL, Truitt W, Fitz SD, Minick PE, Dietrich A, Sanghani S et al (2010). A key role for orexin in panic anxiety. Nat Med 16: 111-115.

Johnston AL, File SE (1989). Yohimbine's anxiogenic action: evidence for noradrenergic and dopaminergic sites. Pharmacol Biochem Behav 32: 151-156.

Kehne JH, Cain CK (2010). Therapeutic utility of non-peptidic CRF1 receptor antagonists in anxiety, depression, and stressrelated disorders: evidence from animal models. Pharmacol Ther 128: $460-487$.

Koob G, Kreek MJ (2007). Stress, dysregulation of drug reward pathways, and the transition to drug dependence. $A m \mathrm{~J}$ Psychiatry 164: 1149-1159.

Kupferschmidt DA, Tribe E, Erb S (2009). Effects of repeated yohimbine on the extinction and reinstatement of cocaine seeking. Pharmacol Biochem Behav 91: 473-480.

Laorden ML, Ferenczi S, Pintér-Kübler B, González-Martín LL, Lasheras MC, Kovács KJ et al (2012). Hypothalamic orexin-A neurons are involved in the response of the brain stress system to morphine withdrawal. PLoS One 7: e36871.

Lee B, Tiefenbacher S, Platt DM, Spealman RD (2004). Pharmacological blockade of + 2-adrenoceptors induces reinstatement of cocaine-seeking behavior in squirrel monkeys. Neuropsychopharmacology 29: 686-693.

MacDonald E, Scheinin H, Scheinin M (1988). Behavioural and neurochemical effects of medetomidine, a novel veterinary sedative. Eur J Pharmacol 158: 119-127.

Marinelli P, Funk D, Juzytsch W, Harding S, Rice K, Shaham Y et al (2007). The CRF1 receptor antagonist antalarmin attenuates yohimbine-induced increases in operant alcohol self-administration and reinstatement of alcohol seeking in rats Psychopharmacology 195: 345-355.

Nair SG, Adams-Deutsch T, Epstein DH, Shaham Y (2009). The neuropharmacology of relapse to food seeking: methodology, main findings, and comparison with relapse to drug seeking. Prog Neurobiol 89: 18-45.

Nair SG, Navarre BM, Cifani C, Pickens CL, Bossert JM, Shaham Y (2011). Role of dorsal medial prefrontal cortex dopamine D1family receptors in relapse to high-fat food seeking induced by the anxiogenic drug yohimbine. Neuropsychopharmacology 36: 497-510.
Pepelko WE, Dixon GA (1975). Arterial blood gases in conscious rats exposed to hypoxia, hypercapnia, or both. J Appl Physiol 38: 581-587.

Piccoli L, Micioni Di Bonaventura MV, Cifani C, Costantini VJA, Massagrande M, Montanari D et al (2012). Role of orexin-1 receptor mechanisms on compulsive food consumption in a model of binge eating in female rats. Neuropsychopharmacology 37: 1999-2011.

Pich EM, Lorang M, Yeganeh M, Rodriguez de Fonseca F, Raber J, Koob GF et al (1995). Increase of extracellular corticotropinreleasing factor-like immunoreactivity levels in the amygdala of awake rats during restraint stress and ethanol withdrawal as measured by microdialysis. J Neurosci 15: 5439-5447.

Rassnick S, Heinrichs SC, Britton KT, Koob GF (1993). Microinjection of a corticotropin-releasing factor antagonist into the central nucleus of the amygdala reverses anxiogenic-like effects of ethanol withdrawal. Brain Res 605: 25-32.

Richards J, Simms J, Steensland P, Taha S, Borgland S, Bonci A et al (2008). Inhibition of orexin-1/hypocretin-1 receptors inhibits yohimbine-induced reinstatement of ethanol and sucrose seeking in Long $\Gamma$ ÇôEvans rats. Psychopharmacology 199: 109-117.

Schmidt M, Andrews R, Ark P, Brown T, Mannaert E, Steckler T et al (2010). Dose-dependent effects of the CRF1 receptor antagonist R317573 on regional brain activity in healthy male subjects. Psychopharmacology 208: 109-119.

Seymour PA, Schmidt AW, Schulz DW (2003). The pharmacology of CP-154,526, a non-peptide antagonist of the CRH1 receptor: a review. CNS Drug Rev 9: 57-96.

Shepard JD, Bossert JM, Liu SY, Shaham Y (2004a). The anxiogenic drug yohimbine reinstates methamphetamine seeking in a rat model of drug relapse. Biol Psychiatry 55: 1082-1089.

Shepard JD, Bossert JM, Liu SY, Shaham Y (2004b). The anxiogenic drug yohimbine reinstates methamphetamine seeking in a rat model of drug relapse. Biol Psychiatry 55: 1082-1089.

Shin LM, Liberzon I (2009). The neurocircuitry of fear, stress, and anxiety disorders. Neuropsychopharmacology 35: 169-191.

Simmons AN, Arce E, Lovero KL, Stein MB, Paulus MP (2009). Subchronic SSRI administration reduces insula response during affective anticipation in healthy volunteers. Int J Neuropsychopharmacol 12: 1009-1020.

Singewald N, Salchner P, Sharp T (2003). Induction of c-Fos expression in specific areas of the fear circuitry in rat forebrain by anxiogenic drugs. Biol Psychiatry 53: 275-283.

Van Pett K, Viau V, Bittencourt JC, Chan RKW, Li HY, Arias C et al (2000). Distribution of mRNAs encoding CRF receptors in brain and pituitary of rat and mouse. J Comp Neurol 428: 191-212.

Wada T, Fukuda N (1991). Effects of DN-2327, a new anxiolytic, diazepam and buspirone on exploratory activity of the rat in an elevated plus-maze. Psychopharmacology 104: 444-450.

Wadenberg M-L (1992). Antagonism by 8-OH-DPAT, but not ritanserin, of catalepsy induced by SCH 23390 in the rat. J Neural Transm 89: 49-59.

Zahrt J, Taylor JR, Mathew RG, Arnsten AF (1997). Supranormal stimulation of D1 dopamine receptors in the rodent prefrontal cortex impairs spatial working memory performance. J Neurosci 17: $8528-8535$.

Supplementary Information accompanies the paper on the Neuropsychopharmacology website (http://www.nature.com/npp) 